\section{Survival in mantle cell lymphoma after frontline treatment with R-bendamustine, R-CHOP and the Nordic MCL2 regimen - a real world study on patients diagnosed in Sweden 2007-2017}

The optimal frontline treatment of mantle cell lymphoma (MCL) with respect to long-term survival remains undefined. Intensified immunochemotherapy including rituximab (R), cytarabine and autologous hematopoetic cell transplant (HD-AHCT) upfront, such as the Nordic MCL2 protocol, has demonstrated improved disease control. ${ }^{1-3}$ Elderly patients may benefit from R-CHOP or R-bendamustine (BR), albeit the regimens have not been robustly evaluated in a randomized setting or in observational studies. ${ }^{1,4,5}$ Here, we used the Swedish Lymphoma Register (SLR), a nationwide register initiated in 2000 with a reported coverage of $~ 95 \%$, ${ }^{6}$ in order to investigate overall and relative survival in a population-based cohort of patients diagnosed with MCL between Jan 2007 and Sept 2017. Particularly, we report outcome by given treatment with specific focus on the currently recommended treatment strategies upfront: BR, R-CHOP, the Nordic MCL2 protocol and curative radiotherapy to limited stage disease.

Data on patient characteristics and frontline treatment administered was retrieved from the SLR. Survival status was obtained from the national population register. Cases fulfilling all of the following criteria were categorized as having been treated with curative radiotherapy: stage I-II disease; treatment with single radiotherapy; reported curative intent and radiation dose of 30-40 Gy. Chemotherapy treatment subgroups (MCL2, BR and R$\mathrm{CHOP}$ ) were compared using t-test or $\mathrm{chi}^{2}$-test (continuous or categorical variables). Follow-up began 90 days after diagnosis to allow for treatment completion and ended on date of death (from any cause) or on 20 October 2018, whichever occurred first. Cox regression models were used to estimate hazard ratios (HRs) with 95\% confidence intervals (CIs) comparing all-cause mortality rates between treatment groups. Both uni- and multivariable models were considered, allowing for adjustments for age at diagnosis, calendar year of diagnosis, sex, WHO performance status (PS), white blood cell count (WBC), lactate dehydrogenase (LDH) and the MCL International Prognostic Index (MIPI). Interaction models were fitted with age at diagnosis (dichotomized into $<70 / \geq 70$ years) as an effect modifier. Non-parametric estimates of overall survival (OS) were calculated by age group using the Kaplan-Meier method. Marginal (standardized) OS was estimated using predictions from a flexible parametric survival model. ${ }^{9}$ As a measure of net (cause-specific) survival, non-parametric relative survival (RS) estimates were calculated using the actuarial method and standardized estimates were predicted from a flexible parametric relative survival model corresponding to that in the OS analysis. All statistical analyses were performed using Stata (StatCorp, 2017. Stata Statistical Software: Release 16. College station, TX: StataCorp LLC). The study was approved by the Regional Board of the Ethical Committee in Lund, Sweden (2018/739).

In total, 1277 patients were included in the study. The median age at diagnosis was 71 years, the majority were male $(70 \%)$ and Ann Arbor stage III-IV (80\%) (Table 1). Frontline systemic treatment was reported in 818 patients $(63 \%)$. Among systemic treatments, MCL2 $(\mathrm{n}=268,33 \%), \mathrm{BR}(\mathrm{n}=231,29 \%)$ and R-CHOP $(\mathrm{n}=93$, $12 \%$ ) were the regimens most frequently applied. The number of patients receiving maintenance rituximab was $10(11 \%)$ after R-CHOP, 18 (8\%) after BR and 14 $(5 \%)$ after MCL2. In total, 218 patients received HDAHCT after MCL2 $(82 \%)$ and one $(<1 \%)$ after BR (Online Supplementary Table S1). Patients who received BR and $\mathrm{R}-\mathrm{CHOP}$ were comparable in terms of mean age $(75.5$ and 73.5, $P=0.39$ ) at diagnosis, performance status (WHO PS $0-187 \%$ vs. $82 \%, P=0.32$ ) and high risk MIPI (55\% vs. $49 \%, P=0.36$ ). Patients who were treated with MCL2 had a lower mean age (60 years, $P<0.0001)$, lower PS (WHO PS 0-1 n=251; 94\%, $P=0.01$ (vs. BR, $P<0.0001$; vs. R-CHOP, $P<0.0001)$ ) and were less likely to score a high-risk MIPI $(26 \%$, (vs. R-CHOP, $P=0.02$; vs. BR, $P<0.0001)$ ) compared to $\mathrm{R}-\mathrm{CHOP}$ and $\mathrm{BR}$, respectively. A total of 1182 patients were included in the survival analyses (95 were excluded due to lack of follow-up (FU) within 90 days after diagnosis). In patients receiving any systemic therapy, median OS was 4.9 years (IOR 1.5$\mathrm{NR}$ ) at a median FU time of 3.5 years (IOR 1.4-5.8). Among patients of all ages, treatment with MCL2 was associated with a lower all-cause mortality than BR in univariable models ( $\mathrm{HR}=0.49$ (95\% CI $0.37-0.66)$ ) or when adjusting for MIPI ( $\mathrm{HR}=0.66$ (95\% CI 0.48-0.91)) but not when adjusting for the individual prognostic factors included in MIPI (HR=1.06 (95\% CI 0.71-1.56)). By age stratification $(<70 / \geq 70)$, no differences in all-cause mortality were observed in patients receiving MCL2 and BR. Patients treated with R-CHOP demonstrated higher all-cause mortality rates compared to BR-treated patients in univariable analysis, either including all patients or by age stratification. In multivariable models, no differences in all-cause mortality were observed. Survival proportions by age and treatment groups are presented in Figure 1 and Online supplementary Table S2. In patients $<70$ years, the unadjusted three-year OS was $0.80(95 \%$ CI $0.75-0.85)$ for MCL2, 0.79 (95\% CI 0.62-0.89) for BR, and 0.62 (95\% CI $0.39-0.78)$ for R-CHOP. The corresponding marginal three-year OS was $0.78(95 \% \mathrm{CI}$ $0.74-0.82), 0.75$ (95\% CI $0.67-0.84)$ and 0.62 (95\% CI $0.51-0.76)$. In patients aged $\geq 70$ years, the unadjusted three-year OS was 0.68 (95\% CI $0.60-0.74)$ for BR and 0.49 (95\% CI 0.36-0.61) for R-CHOP and the corresponding marginal three-year OS was 0.55 (95\% CI 0.45 $0.67)$ and 0.40 (95\% CI $0.22-0.71)$. Analysis of RS demonstrated very similar results as the analysis of OS (Online Supplemenetary Figure S1). In patients given curative radiotherapy $(n=26(2 \%)$, median age was 64 years; all had WHO PS 0-1) and five-year OS was 0.75 (95\% CI 0.51-0.88).

This study reports overall and relative survival in an unselected cohort of patients diagnosed with MCL in 2007-2017, receiving frontline treatment with R-CHOP, $\mathrm{BR}$, or the Nordic MCL2 protocol. The results demonstrate no significant difference in overall or relative survival by intensified MCL2 protocol or R-CHOP compared to BR, by adjustment for prognostic factors or in age-stratified analysis. Furthermore, our analysis demonstrates long-term survival with curative radiotherapy in limited stage MCL. R-CHOP and BR patients were comparable whereas MCL2 patients were younger, had better performance status and less frequent MIPI high-risk score, thus the adjusted models were relevant. The lower all-cause mortality after MCL2 compared to BR in a univariable model or by adjustment for MIPI, but not when adjusting for individual prognostic factors or by age stratification, could be explained by the fact that MIPI is largely driven by age and WHO PS. Moreover, MCL2 is seldom considered in patients $\geq 70$ years, thus the results from age stratification are expected to be 
more reliable. The three-year OS in $\mathrm{BR}$ and $\mathrm{R}-\mathrm{CHOP}$ treated patients is similar to the population-based cohort reported by Villa et al. ${ }^{8}$ but lower than after R-CHOP in the European MCL Elderly trial, as may be expected from an unselected cohort. ${ }^{1}$ In the latter trial, maintenance rituximab after R-CHOP was associated with a benefit in OS, which was also demonstrated in younger patients. ${ }^{1,9}$ Unfortunately, we were not able to confirm these data in the real-world setting due to a limited number of cases. As our study cannot prove that BR issuperior to R-CHOP, durther analysis on the effect of mainte- nance rituximab would be valuable. HD-AHCT was established in MCL based on its association with improved OS in a randomized European pre-rituximab trial, ${ }^{10}$ although not confirmed after the addition of rituximab and cytarabine to CHOP-based induction and the use of maintenance with rituximab. ${ }^{3,-11}$ Consequently, HD-AHCT is currently challenged in the ongoing phase III TRIANGLE (NCT02858258) trial. Reviewing the standardized estimates of OS in our analysis, survival after MCL2 and BR may be comparable during FU initial time, as represented by chemo-sensitive cases. The less steep

Table 1. Patient characteristics

\begin{tabular}{|c|c|c|c|c|c|c|c|}
\hline Variable & $\begin{array}{l}\text { All patients } \\
\text { N (col \%) }\end{array}$ & $\begin{array}{l}\text { MCL2 } \\
\text { N }(\operatorname{col} \%)\end{array}$ & $\begin{array}{c}\text { BR } \\
\text { N (col \%) }\end{array}$ & $\begin{array}{l}\text { R-CHOP } \\
\text { N (col \%) }\end{array}$ & $\begin{array}{l}\text { Other } \\
\text { systemic* } \\
N(\text { col \%) }\end{array}$ & $\begin{array}{l}\text { Curative } \\
\text { radiotherapy** } \\
\text { N (col \%) }\end{array}$ & $\begin{array}{l}\text { Other/ } \\
\text { Missing*** } \\
\text { N (col\%) }\end{array}$ \\
\hline Overall (row \%) & $1,277(100)$ & 268 (21) & $231(18)$ & $93(7)$ & $226(18)$ & $26(2)$ & $433(34)$ \\
\hline $\begin{array}{l}\text { Year of diagnosis } \\
2007-2012 \\
2013-2017\end{array}$ & $\begin{array}{l}667(52) \\
610(48)\end{array}$ & $\begin{array}{l}151(56) \\
117(44)\end{array}$ & $\begin{array}{c}81(35) \\
150(65)\end{array}$ & $\begin{array}{l}76(82) \\
17(18)\end{array}$ & $\begin{array}{c}170(75) \\
56(25)\end{array}$ & $\begin{array}{l}18(69) \\
8(31)\end{array}$ & $\begin{array}{l}171(39) \\
262(61)\end{array}$ \\
\hline \multicolumn{8}{|l|}{ Age at diagnosis } \\
\hline Median (IQR) & $71(64-79)$ & $62(56-66)$ & $75(71-80)$ & 74 (69-79) & $76(70-83)$ & $64(59-73)$ & $73(66-81)$ \\
\hline$<50$ & $45(4)$ & $26(10)$ & $0(0)$ & $0(0)$ & $1(0)$ & $2(8)$ & $16(4)$ \\
\hline $50-59$ & 137 (11) & $85(32)$ & $2(1)$ & $3(3)$ & $5(2)$ & $6(23)$ & $37(8)$ \\
\hline $60-69$ & $376(29)$ & $142(53)$ & $39(17)$ & $24(26)$ & $48(21)$ & $11(42)$ & $113(26)$ \\
\hline $70-79$ & $411(32)$ & $15(6)$ & $127(55)$ & $46(49)$ & $75(33)$ & $3(12)$ & $144(33)$ \\
\hline$\geq 80$ & $308(24)$ & $0(0)$ & $63(27)$ & $20(22)$ & $94(42)$ & $4(15)$ & $125(29)$ \\
\hline $\begin{array}{l}\text { Sex } \\
\quad \text { Male } \\
\text { Female }\end{array}$ & $\begin{array}{l}912(71) \\
365(29)\end{array}$ & $\begin{array}{c}202(75) \\
66(25)\end{array}$ & $\begin{array}{l}161(70) \\
70(30)\end{array}$ & $\begin{array}{l}67(72) \\
26(28)\end{array}$ & $\begin{array}{c}167(74) \\
59(26)\end{array}$ & $\begin{array}{c}19(73) \\
7(27)\end{array}$ & $\begin{array}{l}296(68) \\
137(32)\end{array}$ \\
\hline \multicolumn{8}{|l|}{ Ann Arbor stage } \\
\hline I & $70(5)$ & $5(2)$ & $2(1)$ & $3(3)$ & $7(3)$ & $15(58)$ & $38(9)$ \\
\hline II & $124(10)$ & $18(7)$ & $26(11)$ & $8(9)$ & $19(8)$ & $11(42)$ & $42(9)$ \\
\hline III & $144(11)$ & $30(11)$ & $28(12)$ & $15(16)$ & $34(15)$ & $(0)$ & $37(9)$ \\
\hline IV & $885(69)$ & $212(79)$ & $166(72)$ & $65(70)$ & $154(68)$ & $(0)$ & $288(62)$ \\
\hline Missing & $54(4)$ & $3(1)$ & $9(4)$ & $2(2)$ & $12(5)$ & $2(8)$ & $28(6)$ \\
\hline $\begin{array}{l}\text { MIPI } \\
\text { Median }(\mathrm{IQR}) \\
\text { Low }(<5.7) \\
\text { Intermediate }(5.7-6.1) \\
\text { High }(\geq 6.1) \\
\quad \text { Missing }\end{array}$ & $\begin{array}{l}6.3(5.9-6.8) \\
147(12) \\
323(25) \\
573(45) \\
234(18)\end{array}$ & $\begin{array}{l}5.9(5.6-6.3) \\
70(26) \\
84(31) \\
69(26) \\
45(17)\end{array}$ & $\begin{array}{c}6.4(6.1-6.9) \\
7(3) \\
64(28) \\
128(55) \\
32(14)\end{array}$ & $\begin{array}{l}6.5(6.1-6.9) \\
2(2) \\
20(22) \\
44(47) \\
27(29)\end{array}$ & $\begin{array}{l}6.5(6.2-7.1) \\
7(2) \\
36(16) \\
126(55) \\
57(26)\end{array}$ & $\begin{array}{l}5.9(5.4-6.2) \\
6(23) \\
4(15) \\
5(19) \\
9(35)\end{array}$ & $\begin{array}{c}6.3(5.9-6.9) \\
57(13) \\
115(26) \\
199(49) \\
65(14)\end{array}$ \\
\hline \multicolumn{8}{|l|}{ LDH } \\
\hline $\begin{array}{l}\text { Normal } \\
\text { Elevated } \\
\text { Missing }\end{array}$ & $\begin{array}{c}714(56) \\
523(41) \\
40(3)\end{array}$ & $\begin{array}{c}117(44) \\
147(55) \\
4(1)\end{array}$ & $\begin{array}{c}148(64) \\
79(34) \\
4(2)\end{array}$ & $\begin{array}{c}40(43) \\
51(55) \\
2(2)\end{array}$ & $\begin{array}{c}127(56) \\
94(42) \\
5(2)\end{array}$ & $\begin{array}{l}24(9) \\
1(4) \\
1(4)\end{array}$ & $\begin{array}{c}25860) \\
151(35) \\
24(5)\end{array}$ \\
\hline $\begin{array}{l}\text { WHO PS } \\
0-1 \\
2-4 \\
\text { Missing }\end{array}$ & $\begin{array}{c}1,070(84) \\
186(15) \\
21(2)\end{array}$ & $\begin{array}{l}251(93) \\
15(6) \\
2(1)\end{array}$ & $\begin{array}{c}200(87) \\
26(11) \\
5(2)\end{array}$ & $\begin{array}{l}76(82) \\
15(16) \\
2(2)\end{array}$ & $\begin{array}{l}169(75) \\
54(24) \\
3(1)\end{array}$ & $\begin{array}{c}26(100) \\
0(0) \\
0(0)\end{array}$ & $\begin{array}{c}348(80) \\
76(18) \\
7(2)\end{array}$ \\
\hline \multicolumn{8}{|l|}{ WBC } \\
\hline $\begin{array}{l}\text { Normal }(<9 \times 109 / \mathrm{L}) \\
\text { Elevated } \\
\text { Missing }\end{array}$ & $\begin{array}{l}580(45) \\
503(39) \\
194(15)\end{array}$ & $\begin{array}{l}131(49) \\
99(37) \\
38(11) \\
\end{array}$ & $\begin{array}{l}103(45) \\
103(45) \\
25(11) \\
\end{array}$ & $\begin{array}{l}35(38) \\
33(35) \\
25(27)\end{array}$ & $\begin{array}{l}91(40) \\
79(37) \\
51(24)\end{array}$ & $\begin{array}{l}13(50) \\
4(15) \\
9(35)\end{array}$ & $\begin{array}{l}207(48) \\
185(42) \\
46(10)\end{array}$ \\
\hline
\end{tabular}

* Includes treatment with BAC (bendamustine, cytarabine), chlorambucil, CHOP/cytarabine, cyclophosphamide, cytarabine, CVP (cyclophosphamide, vincristine, prednisone), CVIP (cyclophosphamide, etoposide, idarubicin, prednisone), FC (fludarabine, cytarabine), ibrutinib+bendamustine idarubicin ixoten, lenalidomide+bendamustine.Among these, the most frequently reported regimens were chlorambucil $(\mathrm{n}=69)$ and CHOP/cytarabine $(\mathrm{n}=59) .{ }^{*}$ Includes patients with AA stage I-II MCL, treated with single radiotherapy with curative intent, radiation dose $30-40 \mathrm{~Gy}{ }^{* * *}$ Other/missing includes no treatment given, treatment given but not specified, missing information on treatment and non-curative radiotherapy. MCL: mantle-cell lymphoma; R: Rituximab; CHOP: cyclophosphamide, doxorubicin, vincristine, prednisone; MIPI: MCL International Prognostic Index; N: number; col: column;WBC: white blood cell count;WHO PS:World Health Organization Performance Status. Due to rounding, not all percentages add up to 100 


\section{Kaplan-Meier survival estimates}
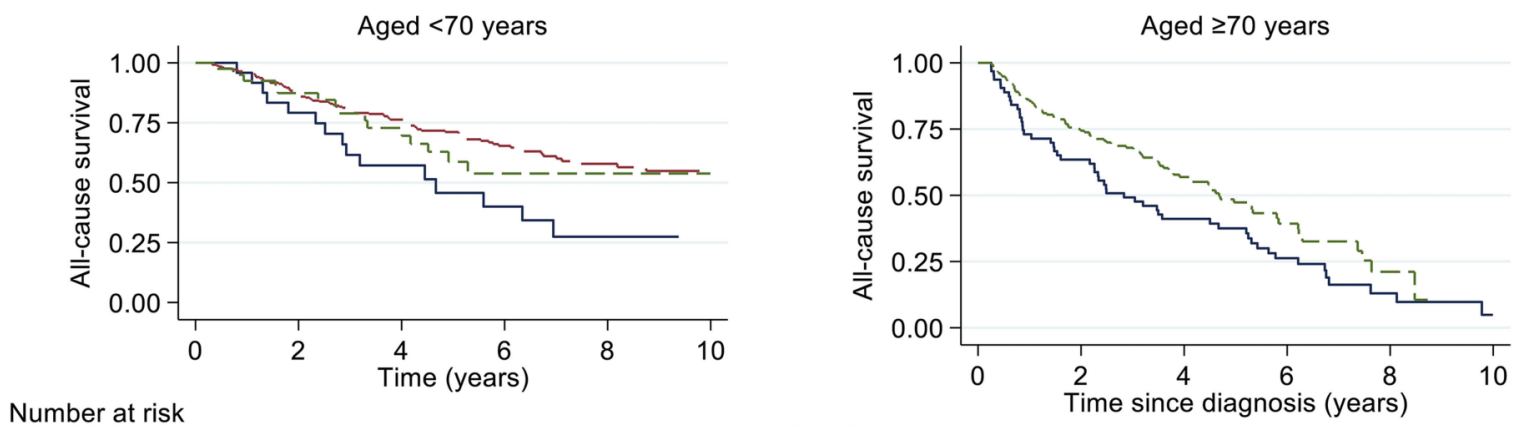

$\begin{array}{rccccc}\text { Number at risk } & & & & & \\ \text { R-CHOP 24 } & 19 & 11 & 7 & 2 & 0 \\ \text { MCL-2/3 252 } & 208 & 155 & 90 & 46 & 0 \\ \text { R-Bendamustine 40 } & 32 & 22 & 8 & 2 & 0\end{array}$

Number at risk

R-Bendamustine 171

$\begin{array}{ccccc}40 & 23 & 12 & 4 & 0 \\ 120 & 64 & 19 & 2 & 0\end{array}$

Standardised OS estimates
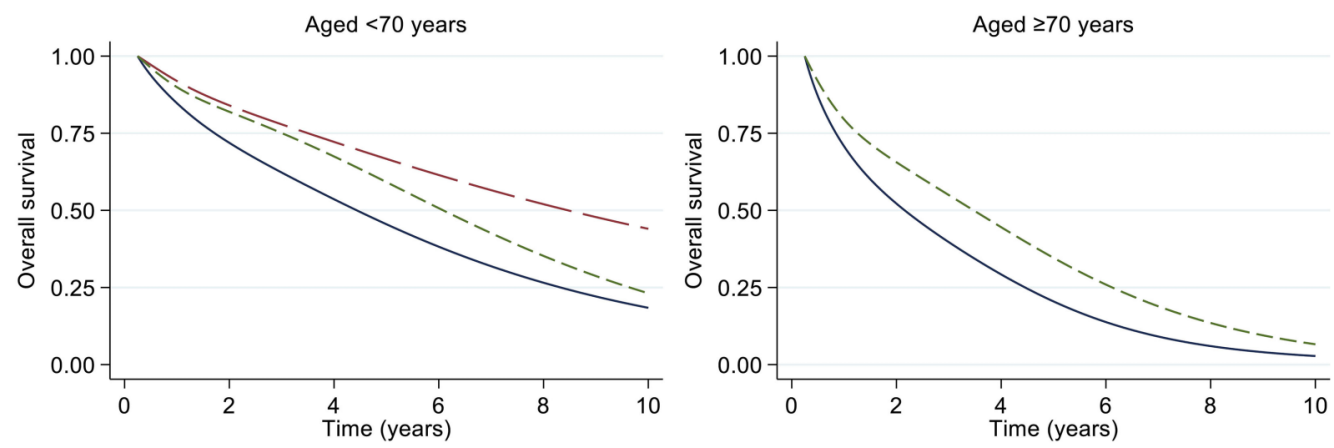

\section{$\mathrm{R}-\mathrm{CHOP} \quad-$ - MCL-2/3}

R-Bendamustine

Figure 1. Unadjusted (top panel) and standardized (bottom panel) overall survival among MCL patients diagnosed in Sweden between 2007 and 2017 , by frontline treatment (BR, R-CHOP, MCL2) and age at diagnosis ( $<70$ and $\geq 70$ years). Standardization was performed over year of diagnosis, sex, age at diagnosis, and performance status (separately for age groups). In patients $\geq 70$ years, MCL2 is not presented due to the limited number of cases. OS: overall survival; R: Rituximab; CHOP: cyclophosphamide, doxorubicin, vincristine, prednisone; MCL2/3: the Nordic MCL2 protocol (R-alternating maxi CHOP/cytarabine with consolidative high-dose chemotherapy with autologous hematopoetic cell transplant). Number at risk table denotes selected time points of estimates (0.3; 2 ; 4; 6; 8; 10 years).

Table 2. Hazard ratio (HRs) with $95 \%$ confidence intervals (Cl) comparing all-cause mortality in relation to given frontline treatment among all patients (top panel) and by age ( $<70$ and $\geq 70$ years) (bottom panel). The analysis included univariable (a) and multivariable models (be). For categorical variables, female sex, WHO PS 0-1 and MIPI low risk were reference groups, respectively. Age and LDH were treated as continuous variables.

\begin{tabular}{|c|c|c|c|c|}
\hline $\begin{array}{l}\text { Treatment regimen } \\
\text { All (n) }\end{array}$ & $\begin{array}{c}\text { HRa (95\% CI) } \\
756\end{array}$ & $\begin{array}{c}\text { HRb }(95 \% \text { Cl) } \\
595\end{array}$ & $\begin{array}{l}\text { HRe }(95 \% \text { Cl) } \\
607\end{array}$ & HRd (95\% CI) \\
\hline BR & 1.00 & 1.00 & 1.00 & 1.00 \\
\hline MCL2 & $0.49(0.37-0.66)$ & $1.06(0.71-1.56)$ & $0.66(0.48-0.91)$ & $0.71(0.51-0.98)$ \\
\hline R-CHOP & $1.51(1.15-2.06)$ & $1.10(0.73-1.66)$ & $1.07(0.73-1.58)$ & $1.23(0.84-1.80)$ \\
\hline Other systemic & $1.49(1.15-1.91)$ & $1.41(1.02-1.92)$ & $1.37(1.01-1.86)$ & $1.44(1.06-1.95)$ \\
\hline \multicolumn{5}{|l|}{$<70$ yrs } \\
\hline $\mathrm{BR}$ & 1.00 & 1.00 & 1.00 & 1.00 \\
\hline MCL2 & $0.77(0.44-1.33)$ & $0.79(0.42-1.46)$ & $0.88(0.49-1.60)$ & $0.92(0.5-1.67)$ \\
\hline R-CHOP & $1.68(0.82-3.43)$ & $1.23(0.50-3.03)$ & $1.10(0.47-2.58)$ & $1.08(0.46-2.55)$ \\
\hline Other systemic & $1.46(0.78-2.75)$ & $1.78(0.86-3.70)$ & $1.51(0.74-3.08)$ & $1.34(0.65-2.74)$ \\
\hline \multicolumn{5}{|l|}{$\geq 70 \mathrm{yrs}$} \\
\hline BR & 1.00 & 1.00 & 1.00 & 1.00 \\
\hline R-CHOP & $1.56(1.10-2.19)$ & $1.03(0.65-1.64)$ & $1.10(0.72-1.69)$ & $1.33(0.87-2.02)$ \\
\hline Other systemic & $1.56(1.19-2.06)$ & $1.55(1.10-2.18)$ & $1.36(0.97-1.90)$ & $1.49(1.07-2.07)$ \\
\hline
\end{tabular}

a) From Cox regression model adjusted for time since diagnosis (time scale). b) From multivariable Cox regression model adjusted for age, sex,WHO PS, WBC and LDH. c) From multivariable Cox regression model adjusted for sex and MIPI as a continuous variable.d) From multivariable Cox regression model adjusted for sex and MIPI as a categorical variable including high and intermediate risk versus low risk. WHO PS:World Health Organization performance status; WBC: white blood cell count; LDH: lactate dehydrogenase; MIPI: Mantle cell lymphoma International Prognostic Index; HR: high-risk; IMR: intermediate risk. 
curve over time among MCL2 treated patients could possibly be related to a deeper remission and longer time to relapse, as previously demonstrated by the intensified strategy. ${ }^{3,11}$ In any case, the lack of a plateau in any of the curves is probably related to chemo-resistant disease, as previously demonstrated in biologic high-risk MCL. ${ }^{12,13}$ The favorable OS in limited-stage MCL patients receiving curative radiotherapy confirms the efficacy of this strategy. ${ }^{14}$ However, the low number of patients studied, the uncertainty of defining a group based on criteria in a retrospective cohort and the potential influence of confounders, i.e., biologic good prognostic factors, should not be neglected.

A major strength of the work presented is the population-based setting and the large size of the cohort, retrieved from a time period after rituximab was introduced in clinical routine. Moreover, the standardized models in both overall and relative survival models improve the reliability of the results. Limitations were the lack of data on molecular markers, comorbidity, second primary malignancies and other factors with potential impact on treatment choice and mortality ${ }^{15}$ as well as the low number of patients treated with maintenance rituximab. Furthermore, the delayed entry may potentially have excluded cases with the most treatment-resistant disease.

To conclude, this study demonstrates that BR may be comparable to intensified treatment strategies in a proportion of patients with MCL. Awaiting the results from ongoing prospective trials on novel combinatory regimens, future studies should focus on a deeper evaluation of predictive markers in relation to established treatment concepts.

Alexandra Albertsson-Lindblad, ${ }^{1}$ Thorgerdur Palsdottir, ${ }^{2,3}$ Karin E. Smedby, ${ }^{2,4}$ Caroline E. Weibull, ${ }^{2}$ Ingrid Glimelius, ${ }^{2,5}$ and Mats Jerkeman ${ }^{1}$

${ }^{1}$ Division of Oncology, Skane University Hospital, Lund University, Lund; ${ }^{2}$ Department of Medicine Solna, Clinical Epidemiology Division, Karolinska Institutet and Karolinska University Hospital, Stockholm; Department of Medical Epidemiology and Biostatistics, Karolinska Institutet, Stockholm; ${ }^{4}$ Department of Medicine Solna, Division of Hematology, Karolinska University Hospital, Stockholm and ${ }^{5}$ Department of Immunology, Genetics and Pathology, Clinical and Experimental Oncology, Uppsala University and Uppsala Akademiska Hospital, Sweden

Correspondence:

ALEXANDRA ALBERTSSON LINDBLAD-

Alexandra.albertsson_lindblad@med.lu.se

doi:10.3324/haematol.2021.279037

Received: April 22, 2021.

Accepted: November 5, 2021.

Pre-published: November 18, 2021

Disclosures: AAL received grant support from Janssen Pharmaceutical NV as part of a collaboration between Karolinska Institutet and Janssen Pharmaceutical NV; CW and TP are part of a research collaboration between Karolinska Institutet and Janssen Pharmaceutical NV for which Karolinska Institutet has received grant support; IG participated in educational seminars run by Janssen Pharmaceutical NV; KES received a research grant from Janssen, honoraria from Takeda and Celgene; MJ received research support from Janssen Pharmaceutica NV, Celgene, Abbvie, Gilead, Roche and Astra Zeneca and honoraria from Roche, Gilead, BMS, Astra Zeneca, Janssen Genmab and Incyte.

Contributions: $A A L, T P, I G, C E W, K E S, M J$ designed the study; TP, CEW, IG, AAL prepared data; AAL, TP and CEW performed data analysis, tables and figures; AAL, TP, IG, CEW, KES, MJ participated in the analysis and interpretation of results; $A A L$ prepared the draft manuscript; $A A L, T P, I G, C E W, K E S, M J$ critically reviewed the manuscript prior to submission.

Funding: This study was financed partly through the Swedish Cancer Society and partly through a public-private real-world evidence collaboration between Karolinska Institutet and Janssen Pharmaceutical NV.

\section{References}

1. Kluin-Nelemans HC, Hoster E, Hermine O, et al. Treatment of older patients with mantle cell lymphoma (MCL): long-term follow-up of the Randomized European MCL Elderly Trial. J Clin Oncol. 2020;38(3):248-256

2. Geisler CH, Kolstad A, Laurell A, et al. Long-term progressionfree survival of mantle cell lymphoma after intensive front-line immunochemotherapy with in vivo-purged stem cell rescue: a nonrandomized phase 2 multicenter study by the Nordic Lymphoma Group. Blood. 2008;112(7):2687-2693.

3. Hermine $\mathrm{O}$, Hoster E, Walewski J, et al. Addition of high-dose cytarabine to immunochemotherapy before autologous stem-cell transplantation in patients aged 65 years or younger with mantle cell lymphoma (MCL Younger): a randomised, open-label, phase 3 trial of the European Mantle Cell Lymphoma Network. Lancet. 2016;388(10044):565-575.

4. Rummel MJ, Niederle N, Maschmeyer G, et al. Bendamustine plus rituximab versus $\mathrm{CHOP}$ plus rituximab as first-line treatment for patients with indolent and mantle-cell lymphomas: an openlabel, multicentre, randomised, phase 3 non-inferiority trial. Lancet 2013;381(9873):1203-1210.

5. Flinn IW, van der Jagt R, Kahl BS, et al. Randomized trial of bendamustine-rituximab or R-CHOP/R-CVP in first-line treatment of indolent NHL or MCL: the BRIGHT study. Blood. 2014;123(19):2944-2952.

6. Cancercentrum i samverkan R. Nationella kvalitetsregistret för lymfom - Årsrapport från Nationell kvalitetsregistret för lymfom, diagnosperioden 2000-2019. 2020. https://cancercentrum.se/globalassets/cancerdiagnoser/blod-lymfom-myelom/lymfom/rapporter/lymfomarsrapport2000-2019.pdf (accessed 16 February 2021).

7. Royston P, Lambert PC. Flexible parametric survival analysis using stata: beyond the Cox model. College Station, TX. Stata Press. StataCorp LP; 2011.

8. Villa D, Sehn LH, Savage KJ, et al. Bendamustine and rituximab as induction therapy in both transplant-eligible and -ineligible patients with mantle cell lymphoma. Blood Adv. 2020;4(15):34863494.

9. Le Gouill S, Thieblemont C, Oberic L, et al. Rituximab after autologous stem-cell transplantation in mantle-cell lymphoma. N Engl J Med. 2017;377(13):1250-1260.

10. Zoellner AK, Unterhalt M, Stilgenbauer S, et al. Long-term survival of patients with mantle cell lymphoma after autologous haematopoietic stem-cell transplantation in first remission: a post-hoc analysis of an open-label, multicentre, randomised, phase 3 trial. Lancet Haematol. 2021;8(9):e648-e657.

11. Gerson JN, Handorf E, Villa D, et al. Survival outcomes of younger patients with mantle cell lymphoma treated in the Rituximab era. J Clin Oncol. 2019;37(6):471-480.

12. Delfau-Larue M-H, Klapper W, Berger F, et al. High-dose cytarabine does not overcome the adverse prognostic value of CDKN2A and TP53 deletions in mantle cell lymphoma. Blood. 2015;126(5):604-611.

13. Eskelund CW, Dahl C, Hansen JW, et al. TP53 mutations identify younger mantle cell lymphoma patients who do not benefit from intensive chemoimmunotherapy. Blood. 2017;130(17):1903-1910.

14. Bernard M, Tsang RW, Le LW, et al. Limited-stage mantle cell lymphoma: treatment outcomes at the Princess Margaret Hospital. Leuk Lymphoma. 2013;54(2):261-267.

15. Glimelius I, Smedby KE, Albertsson-Lindblad A, et al. Unmarried or less-educated patients with mantle cell lymphoma are less likely to undergo a transplant, leading to lower survival. Blood Adv. 2021;5(6):1638-1647 